\title{
GENETICS
}

\section{Divergence shapes recurrence}

Medulloblastoma, one of the most common types of paediatric cancer, is nearly always fatal in its recurrent form. Preclinical models of this disease are based on untreated tumours and, therefore, trials for new agents to treat recurrent medulloblastoma are designed assuming that tumour features are similar at disease presentation and at recurrence; however, the results of a new study by Morrissy and colleagues demonstrate that these forms of medulloblastoma are highly genetically divergent.

Their initial experiments were conducted on transgenic mice with metastatic medulloblastoma that were subject to tumour resection followed by craniospinal irradiation, the standard therapy for children with medulloblatoma. Genetic analysis showed a lack of overlap between the profile of primary tumours and that of matched samples of local or metastatic recurrence. Wholegenome sequencing comparison of therapy-naive samples from patients with medulloblastoma $(n=43)$ with patient-matched samples of recurrence tumours also revealed a high degree of genetic divergence.
Further analysis of the genetic alterations identified in patients' samples indicated that potential drug targets present in primary tumours had disappeared at recurrence. In other words, the evolutionary pressure of surgery and irradiation had selected highly divergent clones that would be resistant to therapy usually used for recurrent disease. In this scenario, a combination therapy approach might help minimize resistance.

Morrissy and colleagues provide evidence of the genetic plasticity of medulloblastoma. They conclude that, in this disease, the therapeutic strategy of targeting truncal mutations that are present in primary samples, but frequently disappear at recurrence, is indicative of failure. Instead, they propose that a biopsy after recurrence should be mandatory for all future clinical trials for medulloblastoma to determine whether the therapeutic targets are present in the dominant clones at recurrence.

Diana Romero

ORIGINAL ARTICLE Morrissy, A. S. et al. Divergent clonal selection dominates medulloblastoma at recurrence. Nature $\mathbf{5 2 9}$, 351-357 (2016) 